\title{
Liver Transplantation in Adults
}

\author{
David H. Van Thiel, Robert R. Schade, Thomas E. Starzl, Shunzaburo Iwatsuki, \\ Byers W. Shaw, JR., Judith S. Gavaler, and Marcus Dugas
}

\author{
Departments of Medicine and Surgery, University of Pittsburgh School of Medicine, \\ Pittsburgh, Pennsylvania 15261
}

\begin{abstract}
Human liver transplantation has been possible since 1967. We report our experience in 32 adult patients who received liver transplants at the University of Pittsburgh over a 16-month period. Survival data, method utilized for patient selection, costs, and morbidity of the procedure are discussed.
\end{abstract}

Orthotopic liver transplantation for humans with advanced liver disease has been possible since 1967 when the first extended survival of such a patient was reported. Since then, more than 400 procedures have been performed; the majority (360) were performed by two groups; one in London (Calne) and the other in the United States (Starzl). At the time of this writing, 237 orthotopic liver transplants have been performed by Starzl; 170 at the University of Colorado and 67 at the University of Pittsburgh. We report the results in adults at the University of Pittsburgh and discuss patient selection, morbidity, cost, and institutional commitment.

\section{PATIENT SELECTION}

From February 1, 1981 through May 30, 1982, 32 orthotopic liver transplants were performed in adults at the University of Pittsburgh School of Medicine; the indications are shown in Table 1. The major indication, accounting for nearly one-third of the cases, was postnecrotic cirrhosis. The second and third most common indications for liver transplantation were advanced primary biliary cirrhosis and hepatocellular carcinoma respectively.

The 32 patients were selected from 115 individuals who were evaluated for liver transplantation. All were referred specifically for consideration for possible liver transplantation. Sixty-eight were considered acceptable candidates based upon the severity of liver disease and the absence of factors known to contradict transplantation.

The disease categories of the potential candidates is shown in Table 2 . Of the 68 acceptable candidates, only one refused transplantation after having been accepted for this procedure.

This work was supported in part by a grant from the Hunt Foundation.

Dr. Van Thiel is the recipient of a Career Development Award from the NIMH AA00016.

Address reprint requests to: David H. Van Thiel, M.D., 1000-J Scaife Hall, Department of Medicine, University of Pittsburgh, School of Medicine, Pittsburgh, Pennsylvania 15261.
Acceptance for transplantation was determined by consensus of the Transplantation Committee. The committee meets weekly and discusses each patient only after completion of an in-hospital evaluation. The committee also reviews potential candidates, discusses the course of each patient in hospital, and receives periodic reports concerning previously transplanted patients who are not in the hospital. Outpatient data are provided by a follow-up nurse coordinator and various physicians who are actively caring for these individuals elsewhere.

The Transplantation Committee is composed of 23 interested individuals who are actively involved in the transplantation program or who serve as concerned individuals with unique backgrounds which could be of importance in the decision-making process. Its specific composition is shown in Table 3. Its meetings are open and can be attended by any interested party within the Health Center. Records of the meetings, discussions, and decisions are maintained for periodic review by the committee, individual members, or other interested individuals.

The number of patients and the reasons for rejecting referred patients for liver transplantation are shown in Table 4. The major reasons for rejecting patients for possible transplantation were that they were not sick enough with their primary hepatic disease, or had metastatic hepatocellular or cholangiolar carcinoma beyond the limits of the liver. Only three patients were refused surgery because they were "too ill." One man had $\alpha_{1}$ antitrypsin deficiency with advanced pulmonary and renal disease, compounded by advanced pancreatitis and pancreatic insufficiency. Another was over 55 years old and had insulin-dependent diabetes mellitus, moderate coexistent renal disease, and active alcohol abuse. The third had $\alpha_{1}$-antitrypsin deficiency, cirrhosis, and active alcoholism.

Not all patients accepted for liver transplantation were transplanted. At present, 18 adult patients have been accepted by the committee and await availability of an appropriate donor organ. From this pool and over the 
TABLE 1. INDICATIONS FOR LIVER Transplantation

\begin{tabular}{|c|c|c|}
\hline & & $\begin{array}{c}\text { No. of } \\
\text { patjents }\end{array}$ \\
\hline Postnecrotic cirrhosis & & $10^{a}$ \\
\hline Primary biliary cirrhosis & & 5 \\
\hline Hepatocellular carcinoma & & 5 \\
\hline$a_{1}$-antitrypsin deficiency & & 3 \\
\hline Cholangiolar carcinoma & & 2 \\
\hline Congenital hepatic fibrosis with Caroli's disease & & 2 \\
\hline Sclerosing cholangitis & & 2 \\
\hline Chronic active hepatitis (Lupoid type) & & 2 \\
\hline \multirow[t]{2}{*}{ Multiple hepatic adenoma } & & 1 \\
\hline & Total & 32 \\
\hline
\end{tabular}

- Three were $\mathrm{HBsAg}^{+}$at the time of transplantation.

Table 2. Hepatic Diseases Present in Acceptable Transplant Candidates

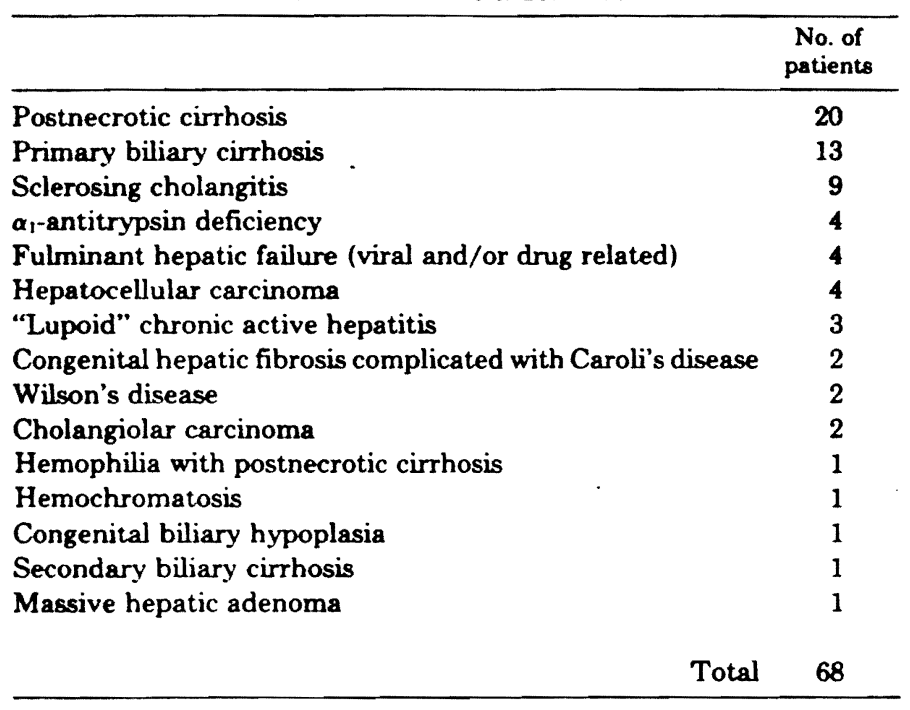

Table 3. The Composition of the liver Transplantation COMMitTeE
5 transplant surgeons
2 gastroenterologists
3 general pediatricians
1 radiologist
1 pathologist
1 anesthesiologist
2 surgical nurses
2 psychiatrists
4 social workers
1 epidemiologist/biostatistician
1 lawyer
23 Total

course of the last 16 months, an additional 18 individuals have died waiting for a transplant (Table 5). The major causes for deaths have been hepatorenal syndrome (11), progressive liver failure (5), sepsis (1), and colonic perforation and sepsis (1).

\section{THE OPERATION}

The initial seven procedures were performed with the donor salvaging team also performing the recipient operation. Recently, this prodigious surgical effort was divided into a donor salvaging team and a recipient trans- plantation team. Such division of surgical effort has resulted in the development of donor procurement skills and recipient transplantation skills by a larger number of individuals and ultimately will enable the program to do more procedures by avoiding the waste of appropriate donor organs because of surgical team exhaustion. Moreover, it is hoped that, by training a larger group of surgeons, our group will spawn groups in other institutions.

The majority of our procedures occur at night. This is because the operation is time-consuming and, by performing it at night, the next morning's schedule of operations is disturbed minimally. Moreover, it allows for the maximum number of accessory individuals such as anesthesiologists, scrub nurses, operating room clerks, and messengers to be available to support the surgical effort without being disturbed by other surgical procedures, except for rare coexistent surgical emergencies. The average anesthesia time for liver transplantation has been $13.5 \pm 0.6 \mathrm{hr}(x \pm$ S.E. $)$.

Three deaths occurred intraoperatively. Two occurred early in our experience and were due to complications related to preoperative portal vein thrombosis. The third occurred as a result of inadequate length of portal vein available for anastomosis after having to take down a prior portal caval shunt. As a result of this limited but tragic experience, we no longer accept candidates for transplantation who have portal vein thrombosis. These patients are identified by the combination of dynamic computerized axial tomography scanning techniques

\section{Table 4. Reasons for Rejecting Patients for Liver} Transplantation

\begin{tabular}{ll}
\hline Not sick enough & 17
\end{tabular}

Hepatic tumor (resectable) 13

Hepatocellular carcinoma with extrahepatic disease 4

Cholangiolar carcinoma with local metastasis 3

Too ill ${ }^{a}$

Portal vein thrombosis

Other therapy available

Alcoholic still drinking

No apparent liver disease

Total 47

\footnotetext{
- One man with $\alpha_{1}$-antitrypsin deficiency, severe emphysema, pan-
} creatic insufficiency, and renal failure; one man $>55$ years, azotemic with insulin-dependent diabetes mellitus, and one woman with an arterial $\mathrm{PO}_{2}<35 \mathrm{~mm} \mathrm{Hg}$ at rest.

Balloon dilatation of extrahepatic biliary tract obstruction relieved the cause of secondary biliary cirrhosis.

Table 5. Patients Who Died Waiting for a Transplant

\begin{tabular}{ll}
\hline Sclerosing cholangitis & 5 \\
Postnecrotic cirrhosis & 3 \\
Fulminant viral hepatitis & $3^{\circ}$ \\
Fulminant drug hepatitis & $2^{\circ}$ \\
$\alpha_{1}$-antitrypsin deficiency & 2 \\
Chronic active hepatitis (lupoid type) & 2 \\
Primary biliary cirrhosis & 1 \\
& Total \\
\hline
\end{tabular}

a Non-A, non-B (1); type B (1), and type A (1).

- Disulfiram (1) and cimetidine (1). 
and/or sonography. In the occasional difficult or uncertain cases, preoperative portal venography is utilized. This latter procedure has an additional risk of producing vein thrombosis and was required in only two cases.

\section{THE IN-HOSPITAL POSTOPERATIVE PERIOD}

The postoperative period is often prolonged and complicated. The time of hospitalization between the transplant operation and discharge averaged $57.1 \pm 7.9$ days. Much of this time was in the surgical intensive care unit (15.0 \pm 3.5 days) immediately after transplantation and after recognition of postoperative complications. The major reasons for surgery following liver transplantation are shown in Table 6. Abscess formation and the need for biliary tract reconstruction comprise the vast majority of postoperative procedures.

The major causes of postoperative disability consist of medical complications which occurred as a result of surgery or during the immediate postoperative period (Table 7). The majority reflect either the effect of renal ischemia or immunosuppression which is required to prevent graft rejection. Three complications were potentially avoidable with appropriate attention to positioning and care of the patient on the operating table (foot drop), surgical detail concerning the need for phrenic nerve dissection and diaphragmatic injury (respiratory insufficiency), and appropriate care and placement of preoperative i.v. lines.

\section{THE OUT-OF-HOSPITAL POSTOPERATIVE PERIOD}

All surviving transplant patients were followed locally for 2 to 6 weeks after discharge to guarantee that immunosuppression was adequate and that no unexpected complications occurred after their return to their own communities and physicians. Each patient was seen weekly and adjustments in diet, immunosuppression, and overall medical management were made.

\section{INSTITUTIONAL COMMITMENTS}

Hepatic transplantation should not be taken lightly simply because a surgeon with the appropriate skills is present in the environment. Such a program can succeed only if the institution supporting the program has made a firm commitment to the success of the program. Such institutional commitment includes adequate amounts of the following:

(1) Intensive care unit beds.

(2) Operating room facilities and personnel available at short notice.

(3) Anesthesia support.

(4) In-hospital beds and outpatient follow-up facilities.

(5) Medical and nursing supporting staff.

(6) Psychiatric support.

(7) Social service support.

(8) Donor procurement program.

(9) Blood product support system.

(10) Family support.

These institutional commitments are essential because, at any one time, between two and four intensive care unit beds can be occupied by transplantation patients and an additional 10 to 20 hospital beds can be occupied by patients at various stages of postoperative
Table 6. Reasons for Posttransplant Surgery

\begin{tabular}{|c|c|}
\hline & No. of procedures \\
\hline Repair of biliary anastomosis & 8 \\
\hline Drainage of abscess & 6 \\
\hline Control of operative bleeding & 3 \\
\hline Control of late gastrointestinal bleeding & 3 \\
\hline Repair of arteriocholedochal fistula & 1 \\
\hline Intestinal infarction & 1 \\
\hline \multirow[t]{2}{*}{ Repeat transplantation } & 2 \\
\hline & - $\mathbf{2 4}^{\bullet}$ \\
\hline
\end{tabular}

" These procedures were performed on 17 of 32 patients transplanted.

Table 7. Acute medical Complications following liver Transplantation

\begin{tabular}{|c|c|}
\hline & No. of patients \\
\hline Activation of herpes viral infection & 12 \\
\hline Acute renal failure requiring dialysis & 12 \\
\hline Oral pharyngeal moniliasis & 12 \\
\hline Foot drop (transient) & 7 \\
\hline Acute psychosis & 5 \\
\hline Respiratory insufficiency & 3 \\
\hline Vocal cord paralysis (temporary) & 2 \\
\hline Cytomegalo viral injectior. & 2 \\
\hline Candida meningitis & 1 \\
\hline Aspergillosis (systemic) & 1 \\
\hline \multirow[t]{2}{*}{ Cerebral vascular accident } & 1 \\
\hline & 58 \\
\hline
\end{tabular}

recovery, new patients who are undergoing their pretransplantation evaluation, and those waiting an appropriate donor organ. Similarly, any transplant operation can be as long as 18 to $20 \mathrm{hr}$ and requires sufficient operating room space, personnel with specialized skills, and anesthesiology support. Because these patients are inevitably ill, usually debilitated, and accompanied by other family members who have come a considerable distance, medical, psychiatric, and social services required to support the patients and their family members are considerable and must be readily available. These must include infectious disease, nephrology, and gastroenterology consultants; physical and respiratory therapy units, group and individual psychiatric support programs, and an active social service unit. Most important is the need for an active donor procurement program. Such a program must have individuals who interact with procurement programs in other institutions to identify and obtain appropriate organs $24 \mathrm{hr}$ a day from anywhere within a 3,000-mile radius. The availability of an emergency air transportation system for patient movement and organ recruitment is extremely important for the success of a transplantation program.

\section{IMPORTANCE OF THE PRETRANSPLANT EVALUATION}

As noted earlier, all potential transplant candidates undergo in-house evaluation prior to their presentation to the Transplantation Committee. During this evaluation, the candidates become acclimated to the hospital setting and the medical/surgical transplantation team. Psychiatric problems are identified and resolved whenever possible prior to surgery and immunosuppression, 
thereby preventing or at least reducing the number of peri- and postoperative psychiatric problems. The specific nature of the primary hepatic disease for which the patient was referred is confirmed or corrected. As of this writing, 12 subjects evaluated for transplantation had an incorrect diagnosis at the time of referral. In one case, the misdiagnosis (Wilson's disease instead of drug-induced hepatic failure) was of major importance and had it been recognized earlier, the need for liver transplantation may have been avoided. In others, misdiagnosis had little if any therapeutic meaning, but could have explained the otherwise confusing course of the patient in response to therapy prior to referral for.transplantation $\left(\alpha_{1}\right.$-antitrypsin deficiency instead of Laennec's cirrhosis, hemachromatosis instead of idiopathic postnecrotic cirrhosis, respectively). Two patients who were diagnosed as having sclerosing cholangitis had cholangiolar carcinoma.

More important is that two candidates with advanced secondary biliary cirrhosis were identified who could be managed by means other than transplantation (Table 4). Finally, one patient had an incidental superficial spreading gastric carcinoma and was referred for therapy prior to consideration for transplantation.

\section{COST OF THE PROGRAM}

The cost of the transplantation program is considerable due to the relatively long duration of hospitalization before, immediately following and after transplantation with the additional high cost of intensive care unit, other specialized health delivery care systems, blood and blood products, and obtaining an appropriate donor organ which includes the cost of transportation to harvest the organ, operating room and anesthesia fees accrued at the donor hospital, and overhead costs of the procurement program.

The reasons for prolonged pretransplant hospitalization include the intrinsic nature or severity of the primary disease, inability to find appropriate out-of-hospital lodging, and inability of the candidate to travel to the hospital within a reasonable time should an appropriate organ be identified.

The reasons for prolonged posttransplantation hospitalization include the numerous posttransplantation surgical procedures to which these patients are exposed (Table 6), and medical complications following liver transplantation (Table 7).

The cost in blood products is considerable; a transplantation program can tax the most efficient of blood procurement programs. The average patient requirements for various blood products used as a direct result of the liver transplantation program are shown in Figure 1 . When intra- and postoperative needs are combined, provision of such materials can be difficult. Moreover, the requirement for red blood cells and plasma is essentially intraoperative while the requirement for platelets continues for several days postoperatively.

The cost of the procedure is considerable. The average hospital bill for patients evaluated and treated at our institution was $\$ 30,636.00 \pm \$ 5,783.00(x \pm$ S.E.). Each also accrued physician charges which averaged $\$ 9,800.00$ $\pm \$ 250.00$ (Table 8).

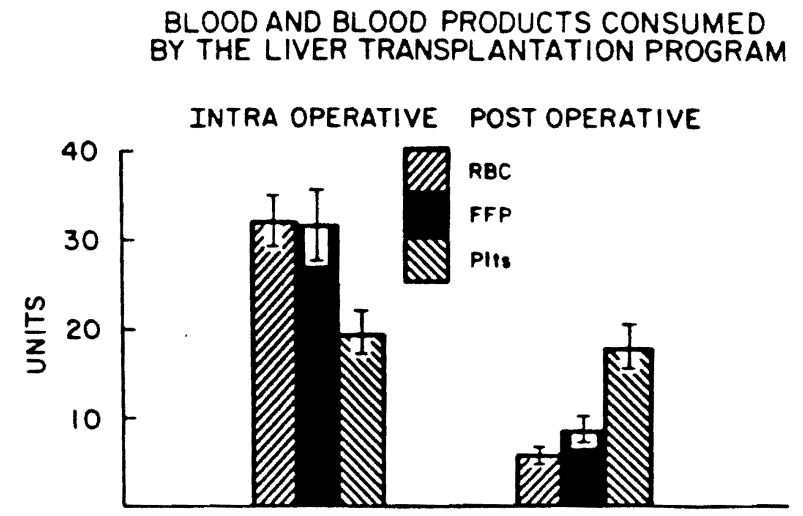

Fig. 1. Blood products utilized during and following liver transplantation. Bars represent means, brackets represent S.E.

Table 8. Physicians' Fees Accrued From a Single Liver Transplantation

\begin{tabular}{lc} 
Surgeon & $\$ 7,000$ (flat fee) \\
Anesthetist & $2,650 \pm 300$ \\
Gastroenterologist & $400 \pm 70$ \\
Nephrologist & $1,200 \pm 200$ \\
Psychiatrist & 85 (flat fee) \\
Other & $300 \pm 300$ \\
\hline
\end{tabular}

\section{SURVIVAL DATA}

Of 32 transplants performed during the study period, 11 recipients died. All but one death occurred within 4 weeks of the surgical procedure. The single late death occurred as a result of bleeding due to a hepatic arterycommon bile duct fistula which could not be repaired. Of the 21 survivors, all but two returned to their homes and reentered an active productive life at their former occupation. Although 11 individuals died as a direct result of liver transplantation ( 3 intraoperatively and 7 in the postoperative period with 1 late death), an additional 18 (Table 5) died waiting to be transplanted. Therefore, the risk of dying waiting for a transplant was 18/68 (26\%) while the risk of dying as a result of the procedure was only slightly greater at $11 / 32(34 \%)$. This latter figure is exaggerated by inclusion of our first four cases who received defective grafts (a start-up complication of a program in a new institution) and the inclusion of three intraoperative deaths in patients who would not presently be considered for transplantation. Survival following successful transplantation is generally excellent and is not associated with continued risk of dying as a result of the primary liver disease. In contrast, the risk of dying of serious liver disease would be expected to increase progressively with increasing duration of untreated illness.

\section{THE FUTURE}

The liver transplantation program at the University of Pittsburgh has several goals to achieve. These are continued technical improvements in the surgical procedure itself; development of better means for preserving donor organs; training of additional surgical and medical teams to perform the procedure, and effective research use of damaged organs to enhance understanding of the disease process and, hopefully, develop methods to prevent or delay the need for transplantation. 